\title{
Déforestation, savanisation et développement agricole des paysages de savanes-forêts dans la zone soudano-guinéenne du Bénin
}

Adi MAMA ${ }^{1,2}$

Issouf BAMBA ${ }^{1}$

Brice SINSIN ${ }^{2}$

Jan BOGAERT ${ }^{3}$

Charles De CANnIÈRE ${ }^{1}$

1 Université libre de Bruxelles, Service d'écologie du paysage et systèmes de production végétale CP 169, 50 Avenue F.D. Roosevelt 1050 Bruxelles

Belgique

2 Université d'Abomey-Calavi Laboratoire d'écologie appliquée 01BP526, Cotonou

Bénin

3 Université de Liège Gembloux Agro-Bio Tech Unité Biodiversité et Paysage 2 Passage des Déportés 5030 Gembloux Belgique

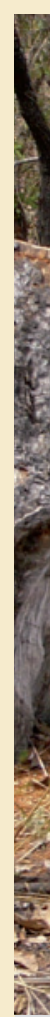

Photo 1.

Exploitation de bois d'œuvre dans les forêts à Bantè. Toutes les espèces de gros diamètre sont coupées, ce qui transforme le paysage forestier en savane. Les savanes sont à leur tour cultivées.

Photo A. Mama, 2006. 


\section{RÉSUMÉ}

\section{DÉFORESTATION, SAVANISATION ET DÉVELOPPEMENT AGRICOLE DES PAYSAGES DE SAVANES-FORÊTS DANS LA ZONE SOUDANO-GUINÉENNE DU BÉNIN}

Les communes de Banté-Glazoué-Ouessé (département des Collines) en zone soudano-guinéenne du Bénin constituent le domaine des mosaïques de forêts et une des principales pourvoyeuses en produits agricoles et en charbon de bois. Ces mosaïques de forêts sont soumises à de multiples formes de dégradation. Une étude de la dynamique spatio-temporelle basée sur une interprétation des images de types Landsat (MSS 1972, TM 1986 et ETM+2006) a été réalisée grâce à l'utilisation du système d'information géographique et des outils d'écologie du paysage afin d'envisager un programme de restauration forestière. Les résultats de cette étude ont permis, grâce à des matrices de transition et des indices de structure spatiale, d'évaluer les mutations liées aux pratiques agricoles. Quatre grands processus de transformation spatiale ont été mis en évidence et quantifiés pour la période de 34 ans (1972 à 2006) retenue pour cette étude : (i) la formation des savanes, (ii) la déforestation, (iii) le développement des activités agricoles et, enfin, (iv) une restauration forestière. La dynamique de la structure spatiale est dominée pendant cette période d'étude par une séquence de création suivie d'agrandissement pour les taches des classes de champs-jachères et de plantations arborées ; d'agrégation pour les classes de savanes et de sols nus-agglomérations ; et de suppression pour les taches de forêt.

Mots-clés : indices structuraux, processus de transformation, déforestation, savanisation, développement agricole, image Landsat, Bénin.

\section{ABSTRACT}

\section{DEFORESTATION, TRANSFORMATION INTO SAVANNAH AND AGRICULTURAL DEVELOPMENT IN THE SAVANNAH AND FOREST LANDSCAPES OF BENIN'S SUDANO-GUINEAN ZONE}

The mosaic forest characterising the municipalities of Banté-Glazoué-Ouessé (Collines district) in Benin's Sudano-Guinean zone is one of the country's main sources of agricultural produce and charcoal. These mosaic forests are being degraded in different ways. A study of their dynamics over space and time, based on interpretations of Landsat-type imagery (MSS 1972, TM 1986 and ETM+ 2006), using a geographic information system and landscape ecology tools, was conducted to support a possible forest restoration programme. The study results were applied, by means of transition matrices and spatial structure indices, to assess changes arising from agricultural practice. Four main spatial transformation processes were identified and quantified over the 34-year period (1972 to 2006) chosen for the study: (i) savannah formation, (ii) deforestation, (iii) development of agricultural activities and, (iv) forest restoration. Over this period, the spatial structure dynamics were dominated by slash-and-burn clearing followed by the enlargement of field and fallow patches and tree plantations, aggregation of savannah, bare soil and urbanised patches and the elimination of forest patches.

Keywords: structural indices, transformation processes, deforestation, transformation into savannah, agricultural development, Landsat imagery, Benin.

\section{RESUMEN}

\author{
DEFORESTACIÓN, SABANIZACIÓN \\ Y DESARROLLO AGRÍCOLA DE PAISAJES \\ DE SABANAS-BOSQUES EN EL ÁREA \\ SUDANO-GUINEANA DE BENÍN
}

Los municipios de Banté-Glazoué-Ouessé (departamento de Collines), en el área sudano-guineana de Benín, configuran el territorio de los mosaicos forestales y son uno de los principales proveedores de productos agrícolas y carbón de leña. Dichos mosaicos están sometidos a múltiples formas de degradación. Se realizó un estudio de la dinámica espaciotemporal para implementar un programa de restauración forestal basándose en la interpretación de imágenes de tipo Landsat (MSS 1972, TM 1986 y ETM+ 2006) y mediante el uso de un SIG y herramientas de ecología del paisaje. Los resultados de este estudio permitieron evaluar las mutaciones vinculadas a las prácticas agrícolas mediante el uso de matrices de transición y de métricas de patrones espaciales. Se evidenciaron y cuantificaron cuatro grandes procesos de transformación espacial en el período de 34 años (1972 a 2006) considerado para este estudio: a) la formación de sabanas, b) la deforestación, c) el desarrollo de actividades agrícolas y, por último, 4) una restauración forestal. La dinámica del patrón espacial está dominada durante este período de estudio por una secuencia de creación seguida de una ampliación de los parches en las clases de campos-barbechos y plantaciones arbóreas; de agregación en las clases de sabanas y suelos desnudosáreas urbanas; y de supresión en los parches de bosques.

Palabras clave: métricas de patrones, procesos de transformación, deforestación, sabanización, desarrollo agrícola, imagen Landsat, Benín. 


\section{Introduction}

L'exploitation abusive des habitats et des ressources biologiques, dans un contexte de faible application des textes réglementaires, constitue la cause principale de la dynamique spatiale des paysages naturels en zone soudanoguinéenne de l'Afrique de l'Ouest (Hountondji, 2008 ; Oloukoi et al., 2006). En effet, aux pratiques agricoles rudimentaires telles que l'abattage et le brûlis des espèces ligneuses lors d'une première mise en culture, s'ajoutent une production soutenue de cultures de rente (le coton, par exemple), une exploitation forestière intense et une pratique incontrôlée des feux de végétation. Ceci résulte des besoins socio-économiques d'une population à forte croissance démographique et d'une urbanisation élevée, entraînant une dégradation de la flore, de la faune et des écosystèmes (IFN, 2007). Au Bénin, la zone soudano-guinéenne constitue une zone d'accueil pour de nombreux migrants agricoles dont les pratiques culturales et les formes d'utilisation des ressources naturelles sont peu respectueuses de l'environnement. Les terres fertiles se font ainsi rares et se limitent actuellement aux zones marécageuses, notamment les bas-fonds.

Cette situation devient une préoccupation majeure, tant pour les institutions scientifiques que pour les gestionnaires des ressources naturelles au Bénin. Étudier la nature et les fondements de ces changements afin de gérer durablement les ressources naturelles suppose un choix d'outils adéquats prenant en compte le caractère spatio-temporel du phénomène. Ainsi, la télédétection se présente comme le moyen le plus approprié pour l'identification et le suivi des changements d'utilisation des sols (Mayaux et al., 2007). En effet, la disponibilité d'images satellitaires depuis les années 1970 et leur fréquence élevée permettent de suivre et de quantifier les modifications du paysage.

En supposant que le développement agricole et ses activités complémentaires (comme par exemple la production du charbon de bois et l'exploitation du bois d'œuvre) sont à la base des modifications du paysage, la présente étude s'est fixé pour objectif principal de déterminer la tendance évolutive de l'occupation des sols sur une période de 34 ans (1972 à 2006) dans trois communes du département des Collines (centre du Bénin), à travers une approche cartographique (télédétection et systèmes d'information géographique) et l'utilisation d'indices de description et de quantification du paysage.

\section{Caractéristiques du secteur d’étude}

La zone d'étude est située au Bénin, entre $8^{\circ}$ et $9^{\circ}$ de latitude Nord et entre $2^{\circ} 10$ et $2^{\circ} 49$ de longitude Est, et couvre une superficie d'environ $7445 \mathrm{~km}^{2}$ (figure 1). Régie par un climat tropical de transition (Afouda, 1990), cette zone se caractérise par des moyennes annuelles de pluviométrie et de température respectives de $1300 \mathrm{~mm}$ et $27^{\circ} \mathrm{C}$. Le relief est une pénéplaine cristalline marquée par la présence d’inselbergs d'une altitude allant de 200 à 400 m (Adam et Boko, 1993). Les sols appartiennent au grand ensemble ferrugineux du milieu tropical (Igue, 2000). L'agriculture est de type extensif, caractérisée par des rendements culturaux faibles. L'exploitation forestière et l'instabilité climatique ont pris de l'ampleur ces dernières années (Oloukoi et al., 2006).

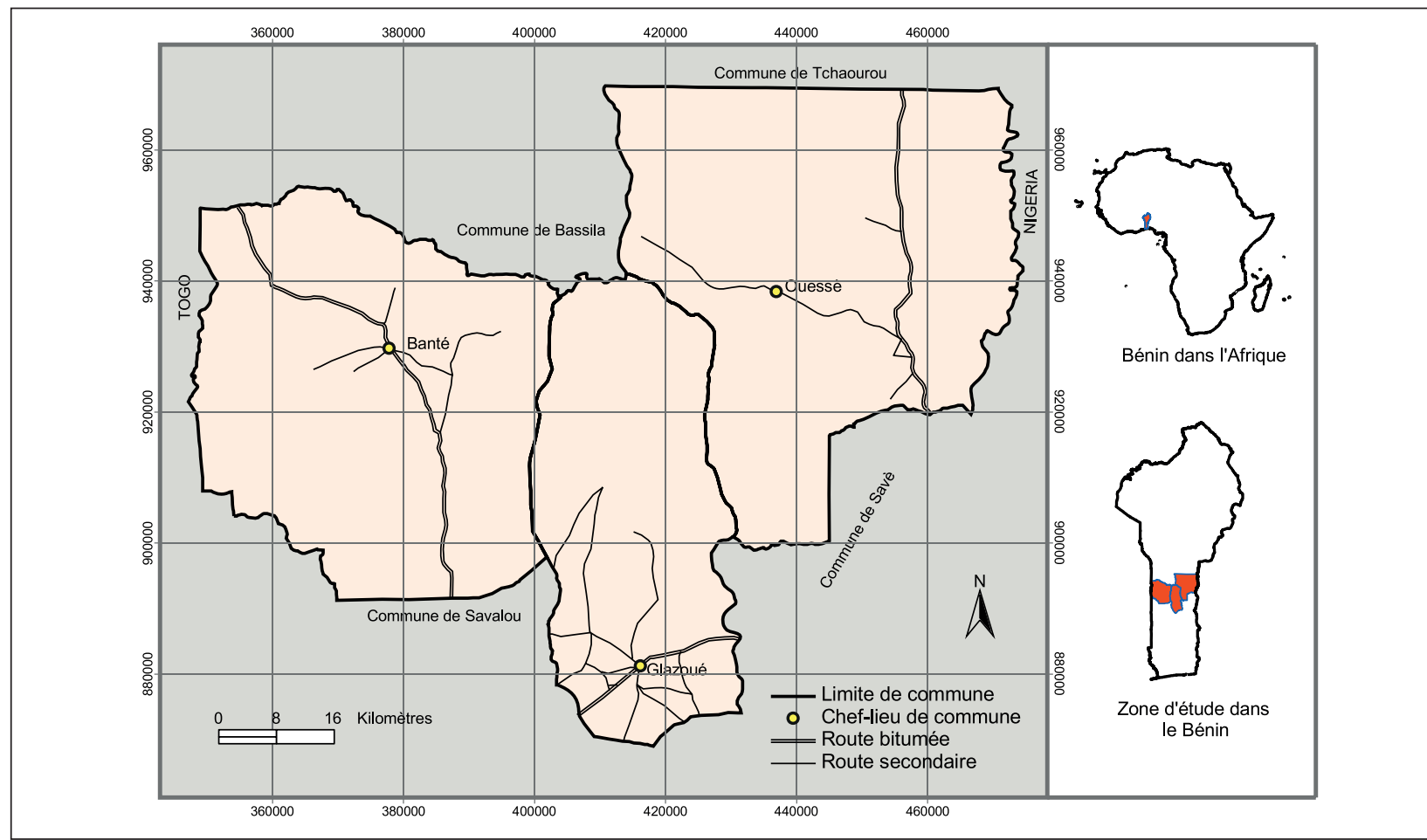

Figure I.

Localisation de la zone d'étude : zone soudano-guinéenne au centre du Bénin dans les communes de Bantè, Glazoué et Ouessè (département des Collines). 


\section{Données et approche méthodologique}

\section{Données et outils}

Une approche cartographique a été adoptée pour l'étude diachronique de la dynamique paysagère à partir de quatre scènes de l'imagerie Landsat dont une MSS (Multi Spectral Scanner) (path 206 et row 054 du 10-11-1972), une TM (Thematic Mapper) (path 192 et row 054 du 13-01-1986), et deux ETM+ (Enhanced Thematic Mapper Plus) (path 192 et row 054 du 12-01-2006 et path 192 et row 055 du 09-012005). Ces images présentent l'avantage d'être disponibles gratuitement et la résolution spatiale de leurs pixels (30 m) permet aussi de mieux identifier les éléments paysagers de notre zone d'étude. Les dates de prise de vue de ces images Landsat correspondent à la saison sèche, ce qui permet d'obtenir des différences spectrales très variées entre les éléments de la couverture végétale. Des sources d'information complémentaires ont été également utilisées afin de faciliter la discrimination des classes d'occupation des sols, notamment pour les images Landsat de 1972 et 1986. II s'agit de la carte topographique (NC-31-II, NC-31-III, NB-31$X X-X X I)$ au 1/200 000 (IGN France, 1969), de documents géographiques anciens de l'administration forestière française de 1960, de la carte de végétation et des données de terrain géoréférencées du Centre national de télédétection et de surveillance du couvert forestier du Bénin de 1978. Le traitement et l'analyse de ces images ont été assurés à l'aide des logiciels ENVI 4.3 et ArcGIS 9.3.

\section{Traitement et analyse des images}

\section{Corrections géométriques, rééchantillonnage et classification des images}

La première étape du traitement des images a consisté en une reprojection des bandes spectrales dans le système UTM (Universal Transverse Mercator) / Zone 31N recouvrant la zone d'étude, et basée sur l'ellipsoïde de référence WGS 84 (World Geodesic System). Afin de corriger la différence de résolution spatiale entre l'image MSS (57 m) et les deux autres images, à savoir l'image TM et celle issue de la mosaïque des deux scènes ETM+, un rééchantillonnage a été effectué. Ce rééchantillonnage effectué dans ENVI 4.3 à partir du module "Resize Data Parameters » a permis de mettre toutes les images à la même résolution spatiale de $30 \mathrm{~m}$. Une composition en fausses couleurs a ensuite été réalisée pour chacune des dates retenues, à partir des trois bandes spectrales 2 (vert), 3 (rouge) et 4 (proche infrarouge) dans l'ordre 4-3-2. Dans l'optique d'améliorer significativement la netteté des images sans trop altérer leur radiométrie (Caloz et al., 1993) afin de faciliter la détection des changements (Tabopda et Fotsing, 2010), une amélioration des contrastes a été également réalisée. Son principe est de redistribuer plus uniformément les valeurs des pixels présentes dans l'image. Par interprétation visuelle (Galicia et Garcia-Romero, 2007), douze types d'occupation des sols ont été identifiés

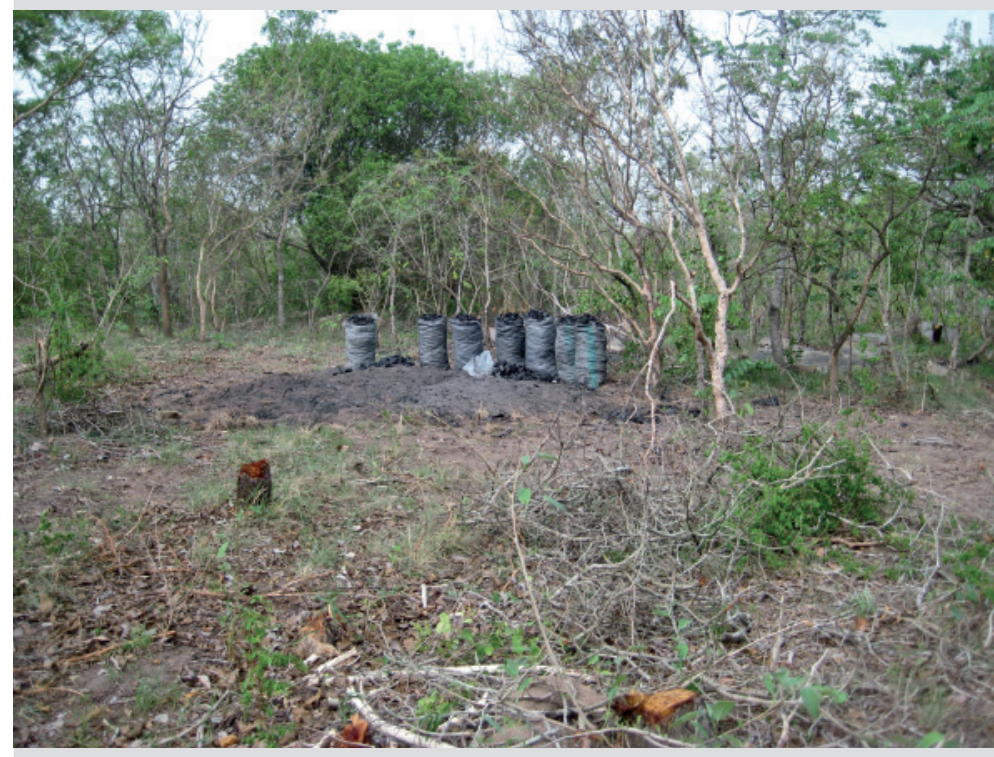

Photo 2.

Carbonisation en pleine forêt à Ouessé. Après la disparition des espèces à gros diamètres ce sont celles à diamètre moyen qui sont coupées et carbonisées.

Photo A. Mama, 2006.

et cartographiés pour chacune des images composites par une approche de classification supervisée. Cette approche consiste à définir des zones d'entraînement sous forme de polygones de pixels sur lesquels s'appuie l'algorithme du maximum de vraisemblance qui calcule la probabilité d'appartenance de chaque pixel à l'une des classes (Mas, 2000). Trois cent cinquante coordonnées géographiques prises directement sur la classification supervisée et encodées dans un GPS ont servi de base pour le contrôle de terrain et les entretiens auprès des populations riveraines.

\section{Évaluation de la précision des classifications}

La précision de la classification a été estimée à l'aide des matrices de confusion. Ainsi, pour chaque classification, des mesures assez communes et largement utilisées telles que la précision globale, le coefficient de Kappa, les erreurs de commission et d'omission (Foody, 2002 ; AbdelKawy et al., 2011) ont été calculées.

\section{Reclassification et filtrage des images}

Les douze classes d'occupation des sols obtenues pendant la classification supervisée ont été reclassées en cinq grandes classes : "forêts ", " savanes ", " champsjachères ", " sols nus-agglomérations » et " plantations arborées ॥. II n'était pas possible de séparer champs et jachères compte tenu de leur ressemblance radiométrique et physionomique pendant la saison sèche. L'application de filtres majoritaires de Kernel à des fenêtres de $8 \times 8$ pixels a permis de résorber les pixels isolés. Le principe du filtrage est de modifier la valeur numérique de chaque pixel en fonction des valeurs des pixels voisins. 


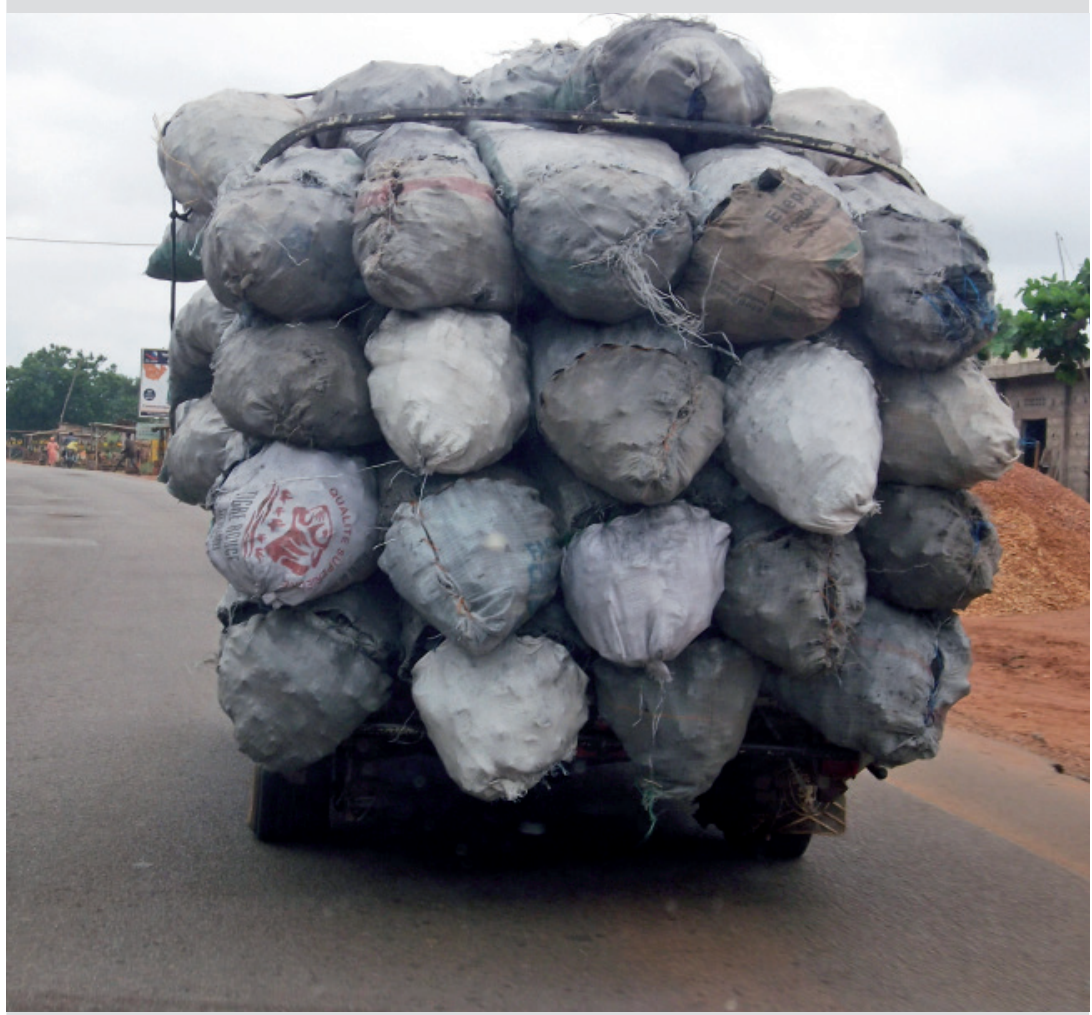

Photo 3.

Transport du charbon de bois vers la ville de Cotonou. Plusieurs véhicules de ce type rentrent chaque jour dans les grandes villes du Sud-Bénin. C'est une activité organisée en filière et bien structurée qui augmente la destruction des formations végétales. Photo A. Mama, 2009.

\section{Résultats}

\section{Qualité des classifications supervisées}

La classification effectuée sur l'image MSS (1972) rééchantillonnée est correcte à $91 \%$ (coefficient de Kappa), avec une précision globale de 93,5\% (tableau I). Les faibles erreurs d'omission et de commission suggèrent que les classes "savanes", « champs-jachères » et « sols nus-agglomérations » sont les mieux classées, c'est-à-dire les moins affectées par les autres classes.

La matrice de confusion de l'image TM de 1986 donne 98,8 \% de précision globale pour un coefficient de Kappa de $99 \%$. Toutes les classes sont majoritairement bien discriminées, avec toutefois quelques légères confusions au niveau des « sols nus-agglomérations ». Enfin, la matrice de confusion de l'image Landsat ETM+ de 2006 révèle que la classification est excellente (Kappa $=97 \%$ ) avec une précision globale de 98,8\%. Les classes "savanes ", " champs-jachères ", " sols nus-agglomérations » et "plantations arborées » sont les mieux classées. Par contre, il existe une certaine confusion entre les classes «forêts » et «plantations arborées ».

\section{Composition de l'occupation des sols à l'échelle du paysage}

L'évolution de l'occupation des sols

\section{Mise en évidence des changements}

Les cartes de changements paysagers ont été obtenues par superposition deux à deux des cartes d'occupation des sols élaborées par classification des images. Les matrices de transition ont été créées pour traduire les fréquences de transition entre les classes au cours d'une période donnée (Robin, 2002 ; Barima et al., 2009). La structure spatiale du paysage a été caractérisée pour chaque classe d'occupation des sols sur la base du nombre de taches (n), de l'aire (a) et du périmètre $(p)$ cumulatifs des taches. L'arbre de décision proposé par Bogaert et al. (2004) a permis de déterminer les dix processus majeurs de transformation spatiale (PTS) des paysages, à savoir l'agrégation, la suppression, la création, la déformation, l'agrandissement, la perforation, le déplacement, le rétrécissement, la fragmentation et la dissection des taches. Les définitions de ces PTS et de l'arbre de décision peuvent être retrouvées dans Bogaert et al. (2004) et Barima et al. (2009). Ainsi, lorsque $n_{1986}>n_{1972}$ ou $\mathrm{n}_{2006}>\mathrm{n}_{1986}$ ou $\mathrm{n}_{2006}>\mathrm{n}_{1972}$, les rapports $t_{\text {observé }}=a_{1986} / a_{1972}, a_{2006} / a_{1986,} a_{2006} / a_{1972}$ ont été comparés à une valeur prédéfinie de $t=0,5$ afin de distinguer la fragmentation et la dissection. Si $t_{\text {observé }}>0,50$, le PTS retenu sera la dissection et si $t_{\text {observé }} \leq 0,50$, le PTS dominant restera la fragmentation. entre 1972 et 2006 fait ressortir de manière générale une diminution de l'étendue des formations forestières (figure 2). En effet, la classe «forêts », qui, en 1972, constituait la matrice dominante du paysage (53\%) (photos 1, 2 et 3 ), a connu une régression spatiale avec une forte tendance à la disparition en 2006. En revanche, les «formations savanicoles " ont connu sur la même période une nette progression, de $38,8 \%$ de la superficie totale de la zone étudiée en 1972 à $76,4 \%$ en 2006 , soit une augmentation annuelle de $2,8 \%$, qui a fait d'elles la matrice dominante du paysage. La superficie totale de la classe " champs-jachères " a connu une augmentation de $6,6 \%$ à 16,8\% entre 1972 et 2006 , soit un taux d'accroissement annuel de 4,5\%.

\section{Changements d'occupation des sols}

En 1986 , seules $15,7 \%$ des $53,5 \%$ des surfaces du paysage occupées par les forêts en 1972 sont restées intactes, tandis que $34,5 \%$ sont devenues des savanes et 3,1 \% ont été totalement converties en espaces non forestiers (tableau II). Les forêts ont ainsi reculé d'environ $35 \%$ en faveur principalement des savanes (31,1\%). La proportion de la classe « champs-jachères » est passée de 6,6 \% à $8,5 \%$ durant la même période. Enfin, la classe « sols nus- 


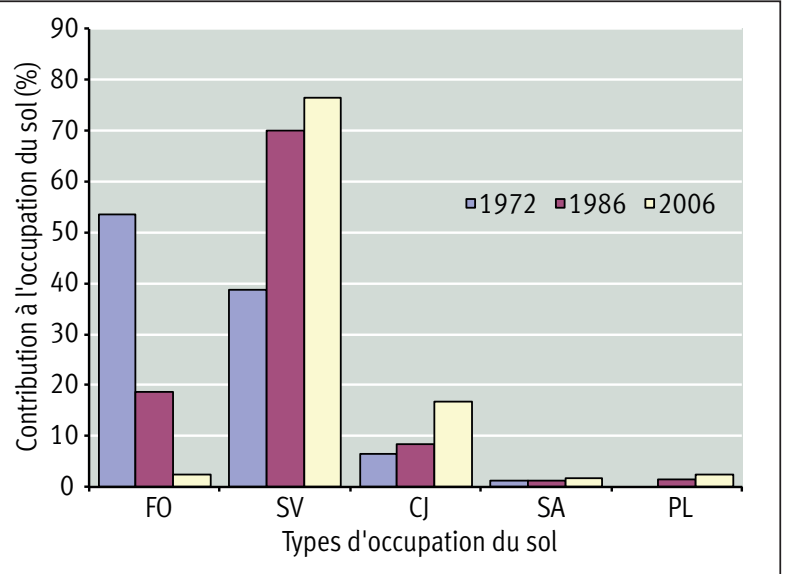

agglomérations », avec 0,6\% de taux de permanence, a constitué la classe la moins stable dans le paysage. De manière globale, cette période 1972-1986 se caractérise par trois principaux types de dynamique paysagère : une stabilité des classes d'occupation des sols $(48,4 \%$ du paysage), une dynamique de densification de la couverture végétale $(7,3 \%$ du paysage), et une ouverture du milieu (44,2\% du paysage) s'exprimant à des degrés divers (forêts vers savanes : 34,6 \% ; forêts vers champs-jachères : $3,1 \%$; savanes vers champs-jachères : $4,3 \%$; savanes vers sols nus-agglomérations et plantations arborées : respectivement $0,2 \%$ et $0,5 \%$; champs-jachères vers sols nusagglomérations et plantations arborées : respectivement $0,5 \%$ et $1 \%)$. Ces dynamiques s'observent également durant la période 1986-2006 mais dans des proportions différentes. En effet, la stabilité des classes d'occupation des sols concerne $62,1 \%$ du paysage contre $6,1 \%$ et $31,8 \%$ respectivement pour la dynamique de densification de la couverture végétale et l'ouverture du milieu. Ainsi, 0,4\%

Figure 2.

Composition de l'occupation des sols en 1972, 1986 et 2006 dans les communes de Bantè, Glazoué et Ouessè (département des Collines) au Bénin. FO = forêts, $\mathrm{SV}=$ savanes, $\mathrm{CJ}=$ champs-jachères, $\mathrm{SA}=$ sols nus agglomérations, $\mathrm{PL}=$ plantations arborées.

Tableau I.

Indices d'évaluation de la précision des classifications supervisées des images Landsat de 1972, 1986 et 2006 dans les communes de Bantè, Glazoué et Ouessè au Bénin.

\begin{tabular}{|c|c|c|c|c|c|c|c|}
\hline & & FO & SV & CJ & SA & PL & Omission (\%) \\
\hline 1972 & Non classifiées & 0,0 & 0,0 & 0,0 & 0,8 & 0,0 & 0,0 \\
\hline & FO & 77,2 & 0,0 & 0,0 & 0,0 & 13,6 & 22,7 \\
\hline & SV & 0,0 & 98,7 & 0,0 & 0,0 & 0,0 & 1,3 \\
\hline & C) & 0,0 & 0,5 & 99,3 & 0,0 & 0,0 & 0,6 \\
\hline & SA & 0,0 & 0,7 & 0,6 & 98,9 & 1,9 & 1,0 \\
\hline & $\mathrm{PL}$ & 22,7 & 0,0 & 0,0 & 0,2 & 84,4 & 15,5 \\
\hline & Commission (\%) & 6,2 & 0,0 & 1,5 & 2,8 & 4,1 & \\
\hline & & & $\begin{array}{l}\text { Précisio } \\
\text { Coefficie }\end{array}$ & $\begin{array}{l}\text { le : } 93 \\
\text { ppa : }\end{array}$ & & & \\
\hline 1986 & Non classifiées & 0,0 & 0,0 & 0,0 & 0,1 & 0,0 & 0,0 \\
\hline & FO & 98,8 & 0,0 & 0,0 & 0,0 & 0,7 & 1,2 \\
\hline & SV & 0,0 & 99,7 & 0,1 & 0,0 & 0,0 & 0,3 \\
\hline & C) & 0,0 & 0,2 & 99,5 & 0,0 & 0,0 & 0,4 \\
\hline & SA & 0,0 & 0,0 & 0,3 & 99,8 & 0,2 & 0,1 \\
\hline & $\mathrm{PL}$ & 1,2 & 0,0 & 0,0 & 0,0 & 99,1 & 0,8 \\
\hline & Commission (\%) & 0,3 & 0,0 & 0,5 & 0,4 & 2,8 & \\
\hline & & & Précisio & le : 99 & & & \\
\hline & & & Coefficie & ppa : & & & \\
\hline 2006 & Non classifiées & 0,0 & 0,0 & 0,0 & 1,0 & 0,0 & 0,0 \\
\hline & FO & 96,8 & 0,0 & 0,0 & 0,0 & 1,7 & 3,2 \\
\hline & SV & 0,0 & 99,0 & 1,3 & 0,0 & 0,0 & 1,0 \\
\hline & C) & 0,0 & 0,9 & 98,0 & 0,0 & 0,0 & 1,9 \\
\hline & $\mathrm{SA}$ & 0,0 & 0,0 & 0,6 & 98,8 & 0,0 & 1,0 \\
\hline & $\mathrm{PL}$ & 3,2 & 0,1 & 0,0 & 0,0 & 98,2 & 1,7 \\
\hline & Commission (\%) & 0,7 & 0,5 & 2,1 & 0,8 & 7,6 & \\
\hline & & & $\begin{array}{l}\text { Précisio } \\
\text { Coefficie }\end{array}$ & $\begin{array}{l}\text { le : } 98 \\
\text { ppa : }\end{array}$ & & & \\
\hline
\end{tabular}


Tableau II.

Matrice de transition de l'occupation des sols (\%) pour les périodes 1972-1986, 1986-2006 et 1972-2006 dans les communes de Bantè, Glazoué et Ouessè (département des Collines) au Bénin.

\begin{tabular}{lrr} 
FO & $\mathbf{1 5 , 7}$ & 34,6 \\
\hline SV & 2,8 & $\mathbf{3 1 , 0}$ \\
\hline CJ & 0,1 & 3,9 \\
\hline SA & 0,1 & 0,4 \\
\hline PL & 0,0 & 0,0 \\
\hline Total & 18,7 & 69,9
\end{tabular}

1986-2006

\begin{tabular}{l|l} 
FO & $\mathbf{0 , 4}$ \\
\hline SV & 1,1 \\
\hline CJ & 0,5 \\
\hline SA & 0,0 \\
\hline PL & 0,7 \\
\hline Total & 2,6
\end{tabular}

1972-2006

\begin{tabular}{ll} 
FO & $\mathbf{1}, 5$ \\
\hline SV & 0,7 \\
\hline CJ & 0,4 \\
\hline SA & 0,0 \\
\hline PL & 0,0 \\
\hline Total & 2,6
\end{tabular}

\begin{tabular}{r|r}
$\mathbf{1 , 5}$ & 50,1 \\
\hline 0,7 & $\mathbf{2 3 , 2}$ \\
\hline 0,4 & 3,0 \\
\hline 0,0 & 0,1 \\
\hline 0,0 & 0,0 \\
\hline 2,6 & 76,4 \\
\hline
\end{tabular}

\begin{tabular}{|r|r|r|}
\hline $\mathbf{0 , 4}$ & 15,7 & 2,7 \\
\hline 1,1 & $\mathbf{5 6 , 9}$ & 10, \\
\hline 0,5 & 3,1 & $\mathbf{3}, 7$ \\
\hline 0,0 & 0,2 & 0, \\
\hline 0,7 & 0,5 & 0,0 \\
\hline 2,6 & 76,4 & 16, \\
\hline
\end{tabular}

\begin{tabular}{l|l} 
CJ & SA \\
\hline 3,1 & 0,0 \\
\hline 4,3 & 0,2 \\
\hline $\mathbf{1 , 1}$ & 0,5 \\
\hline 0,0 & $\mathbf{0 , 6}$ \\
\hline 0,0 & 0,0 \\
\hline 8,5 & 1,3 \\
\hline
\end{tabular}

SA

\begin{tabular}{|c|c|}
\hline PL & Total \\
\hline 0,0 & 53,5 \\
\hline 0,5 & 38,8 \\
\hline 1,0 & 6,6 \\
\hline 0,0 & 1,1 \\
\hline $\mathbf{0 , 0}$ & 0,0 \\
\hline 1,6 & 100 \\
\hline
\end{tabular}

\begin{tabular}{|r|r|}
\hline 2,7 & 0,0 \\
\hline 10,3 & 0,3 \\
\hline 3,7 & 0,6 \\
\hline 0,2 & 0,8 \\
\hline 0,0 & 0,1 \\
\hline 16,8 & 1,7 \\
\hline
\end{tabular}

$\begin{array}{rrr}0,2 & 0,5 & 38,8 \\ 0,5 & 1,0 & 6,6 \\ \mathbf{0 , 6} & 0,0 & 1,1 \\ 0,0 & \mathbf{0 , 0} & 0,0 \\ 1,3 & 1,6 & 100\end{array}$

\begin{tabular}{ll|l}
\hline 0,0 & 0,0 & 18,7
\end{tabular}

$\begin{array}{lll}0,3 & 1,5 & 69,9\end{array}$

$0,6 \quad 0,6 \quad 8,5$

$\begin{array}{lll}\mathbf{0 , 8} & 0,1 & 1,3\end{array}$

$\begin{array}{lll}0,1 & \mathbf{0 , 3} & 1,6 \\ 1,7 & 2,5 & 100\end{array}$

FO = forêts ; SV = savanes ; $\mathrm{CJ}=$ champs-jachères ; $\mathrm{SA}=$ sols nus-agglomérations ; $\mathrm{PL}$ = plantations arborées.

des superficies du paysage occupées par les forêts en 1986 sont restées inchangées en 2006, tandis que 15,7\% de cette portion du paysage ont été converties en classe « savanes » qui devient dominante $(69,9 \%)$, mais aussi la plus stable avec un taux de $56,9 \%$ du paysage.

À l'échelle globale de l'étude (de 1972 à 2006), les forêts ont subi un recul d'environ $52 \%$. En effet, sur les $53,5 \%$ des surfaces occupées par les forêts en 1972, seulement 1,5\% sont restées intactes, 50,1\% sont devenues des savanes et 1,9\% ont été totalement converties en champs-jachères. La proportion des savanes est passée de $38,8 \%$ à $76,4 \%$, soit $96,9 \%$ d'augmentation. Cette classe des savanes devient actuellement la nouvelle matrice d'un paysage antérieurement dominé par la classe des forêts. La même tendance progressive est observée dans la classe des champs-jachères (photo 4) avec un taux de 10,2 \% au détriment des forêts. En effet, sur les 76,4 \% du paysage occupé par les savanes en 2006, 50,1 \% étaient des forêts et $3 \%$ étaient des champs-jachères en 1972. Au cours de cette période, $27,2 \%$ des superficies n'ont pas changé de classe (stabilité), tandis que 4,2 \% des superficies ont évolué en termes de reconstitution végétale à travers un processus de succession. En revanche, $68,6 \%$ des superficies ont subi un changement de classe d'occupation des sols. La savanisation devient ainsi le phénomène le plus important. Elle est suivie par l'extension des champs et jachères.

\section{Dynamique de la structure spatiale}

Entre 1972 et 1986, la classe "forêts » a subi un accroissement du nombre de taches en parallèle à une diminution de l'aire totale (tableau III). II apparaît évident, sur la base de la valeur de $t_{\text {observé }}=0,3$ inférieur au seuil $t=0,5$, que le processus de transformation dominant de cette classe fut la fragmentation. L'augmentation du nombre de taches est associée à une diminution très forte de l'aire de la classe en question. Par contre, la création de nouvelles taches est le processus de transformation dominant dans les classes " savanes ", " champs-jachères » et "sols nus-agglomérations ", avec une augmentation du nombre de taches mais aussi de l'aire totale sur cette période (tableaux III et IV).

Entre les années 1986 et 2006, le processus d'agrégation (fusion des taches) est observé dans les classes " savanes ", "sol nus-agglomérations » et " plantations arborées ", tandis que le processus de création des taches a concerné la classe " champs-jachères ", car l'augmentation du nombre de taches en 2006 s'est accompagnée d'une augmentation de l'aire totale de cette classe. Quant à la classe "forêts ", la diminution du nombre de taches et de l'aire en 2006 par rapport à 1986, avec une augmentation du périmètre, suggère un processus de suppression des taches. 
En résumé, ces processus de transformation spatiale dominants constituent une séquence de création de taches suivie d'un agrandissement (expansion de la taille des taches) pour les classes " champs-jachères » et " plantations arborées ", d'agrégation pour les classes « savanes » et « sols nus-agglomérations », et de suppression des taches de la classe "forêts ».

\section{Discussion}

\section{Approche méthodologique}

L'approche cartographique de l'occupation des sols à travers une série de classifications d'images satellitaires Landsat couplée à une vérité terrain dont les précisions globales ont été hautement appréciables confirme l'importance de la connaissance du milieu et de l'acquisition des données auxiliaires pour l'amélioration de la qualité des traitements d'images (Trepanier et al., 2002). Khorram et al. (1999) et Pham et al. (2007) ont obtenu de faibles valeurs d'exactitude des classifications respectivement de $61 \%$ et de $51 \%$ à cause d'un manque de collecte de points de contrôle et de validation sur le terrain. Bien que nos classifications aient des marges d'erreur statistiquement acceptables, elles ne doivent pas faire perdre de vue les difficultés techniques rencontrées. En effet, l'analyse de certaines parties de l'image a été rendue difficile par la présence de rayures parallèles engendrées par les photodétecteurs. L'image Landsat du 0901-2006 utilisée était la seule disponible pour cette période et présentait des rayures dans la partie ouest du secteur d'étude. Ce dysfonctionnent du capteur Landsat a pu être amoindri grâce à l'existence de documents cartographiques et une bonne connaissance du milieu d'étude. Toutefois, en nous référant aux valeurs de l'indice Kappa, selon les échelles de Pontius (2000), nous pouvons conclure que les résultats de cette analyse sont statistiquement acceptables.

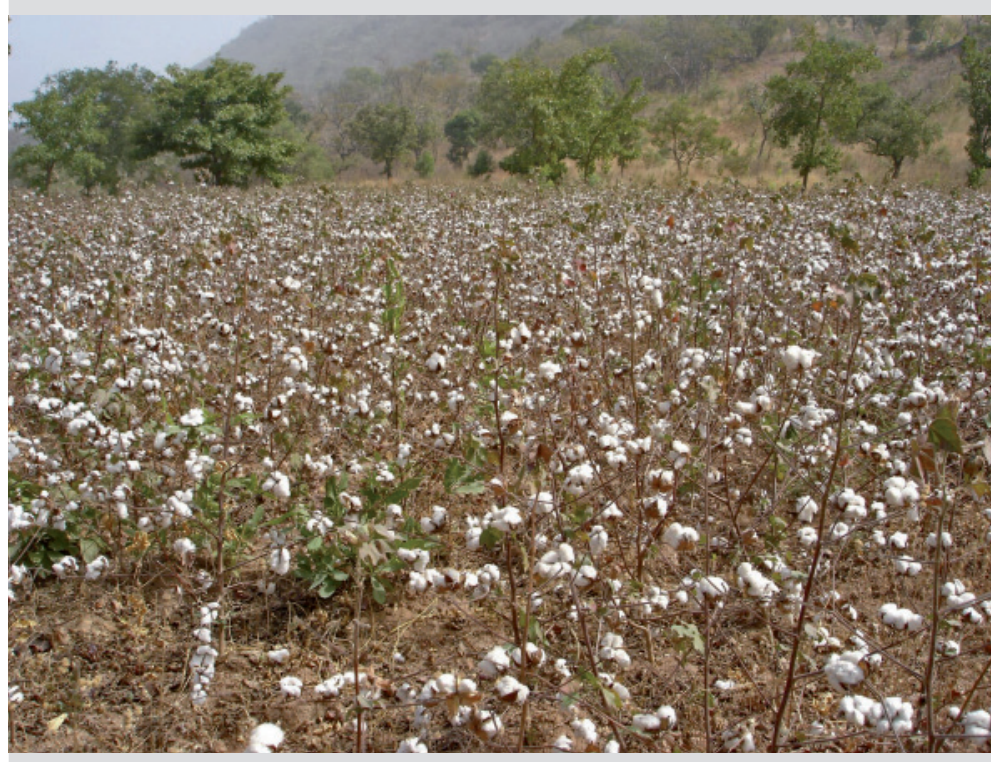

Photo 4.

Champs de coton à Ouessé. En arrière-plan figurent les restes de la végétation avec quelques espèces végétales utiles épargnées car le cotonnier est peu tolérant à l'ombre. Photo A. Mama, 2006.

Cependant, les grandes précisions cartographiques obtenues peuvent aussi dépendre du nombre réduit de classes utilisées et de la définition de parcelles homogènes lors du choix des sites d'entraînement (Caloz et Collet, 2001).

\section{Dynamique de la composition du paysage}

L'effet de l'anthropisation sur la dynamique des paysages forestiers au centre du Bénin se caractérise par une déforestation suivie d'une savanisation. En effet, en 34 ans,

Tableau III.

Nombre $(n)$, aire $(a)$ et périmètre $(p)$ des classes d'occupation des sols en 1972, 1986 et en 2006 dans les communes de Bantè, Glazoué et Ouessè (département des Collines) au Bénin.

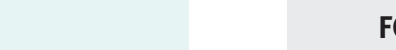

FO

1972

1986

2006

\begin{tabular}{|c|c|c|c|c|c|}
\hline$n_{1972}$ & 51992 & 248619 & 32822 & 39390 & 0 \\
\hline$a_{1972}\left(\mathrm{~km}^{2}\right)$ & 3979,5 & 2890,5 & 491,4 & 84,3 & 0 \\
\hline$p_{1972}(\mathrm{~km})$ & 27483,6 & 305744,5 & 12803,5 & 13257 & 0 \\
\hline
\end{tabular}

\begin{tabular}{|l|c|c|c|c|c|}
\hline$n_{1986}$ & 122936 & 369869 & 37076 & 77281 & 33010 \\
\hline$a_{1986}\left(\mathrm{~km}^{2}\right)$ & 1394,9 & 5205,7 & 631,9 & 97,9 & 115,2 \\
\hline$p_{1986}(\mathrm{~km})$ & 25597 & 171403 & 9055 & 17891,3 & 7052,4 \\
\hline$n_{2006}$ & 43126 & 128677 & 37856 & 24018 & 20933 \\
\hline$a_{2006}\left(\mathrm{~km}^{2}\right)$ & 190,8 & 5686 & 1251,6 & 129,5 & 187,8 \\
\hline$p_{2006}(\mathrm{~km})$ & 29720,1 & 86458,6 & 24434,5 & 7080,4 & 6933,5
\end{tabular}

FO = forêts ; SV = savanes ; CJ = champs-jachères ; SA = sols nus-agglomérations ; PL = plantations arborées. 
Tableau IV.

Identification des processus de transformation spatiale (PTS) des classes d'occupation des sols entre 1972-1986, 1986-2006 et 1972-2006 dans les communes de Bantè, Glazoué et Ouessè (département des Collines) au Bénin à partir de l'arbre de décision de Bogaert et al. (2004) et Barima et al. (2009). Cet arbre de décision définit de façon simplifiée les différents processus de transformation spatiale des classes d’occupation des sols.

\begin{tabular}{lc} 
& $\boldsymbol{n}_{\mathbf{1 9 8 6}}-\boldsymbol{n}_{\mathbf{1 9 7 2}}$ \\
$\mathrm{FO}$ & $\mathbf{7 0 9 4 4}$ \\
\hline $\mathrm{SV}$ & 121250 \\
\hline $\mathrm{CJ}$ & 4254 \\
\hline $\mathrm{SA}$ & 37891 \\
\hline $\mathrm{PL}$ & 33010 \\
& $\boldsymbol{n}_{\mathbf{2 0 0 6}}-\boldsymbol{n}_{\mathbf{1 9 8 6}}$
\end{tabular}

$a_{1986}-a_{1972}$
$-2584,6$
2315,2
140,5
13,6
115,2
$a_{\mathbf{2 0 0 6}}-a_{1986}$

1986-2006

\begin{tabular}{|c|c|c|c|c|}
\hline FO & -79810 & $-1204,1$ & 4123,1 & 0,13 \\
\hline SV & -241192 & 480,3 & $-84944,4$ & 1,09 \\
\hline CJ & 780 & 619,7 & 15379,5 & 1,98 \\
\hline$\overline{S A}$ & -53263 & 31,6 & $-10810,9$ & 1,32 \\
\hline \multirow[t]{2}{*}{$\mathrm{PL}$} & -10810 & 72,6 & $-118,9$ & 1,63 \\
\hline & $n_{2006}-n_{1972}$ & $a_{2006}-a_{1972}$ & $p_{2006}-p_{1972}$ & $a_{2006} / a_{1972}$ \\
\hline FO & -8866 & $-3788,7$ & 2236,5 & 0,05 \\
\hline SV & -119942 & 2795,5 & $-219285,9$ & 0,05 \\
\hline CJ & 50340 & 760,2 & 11631 & 2,55 \\
\hline$\overline{S A}$ & -15372 & 45,2 & $-6176,6$ & 1,54 \\
\hline $\mathrm{PL}$ & 20933 & 187,8 & 6933,6 & 0,00 \\
\hline
\end{tabular}

1972-2006

FO = forêts ; SV = savanes $; \mathrm{CJ}=$ champs-jachères $; \mathrm{SA}=$ sols nus-agglomérations $; \mathrm{PL}=$ plantations arborées. Le nombre de taches, l'aire et le périmètre de chaque classe d'occupation considérée sont calculés en 1972, 1986 et 2006 et désignés par $n$ (nombre de taches), $a$ (aire) et $p$ (périmètre). Les rapports $t_{\text {observé }}=a_{1986} / a_{1972,} a_{2006} / a_{1986,} a_{2006} / a_{1972}$ ont été comparés à une valeur prédéfinie de $t=0,5$ afin de séparer fragmentation et dissection.

la matrice du paysage est passée de «forêts » à « savanes ». Ce changement pourrait s'expliquer par une augmentation de la production de bois de feu, de charbon de bois et des pratiques agricoles (Tchiwanou, 2001). En effet, à défaut d'une diversification des sources d'énergie domestique au Bénin, les essences forestières sont coupées puis carbonisées et vendues dans les grandes agglomérations du pays. Les espèces recherchées sont prioritairement Prosopis africana, Pterocarpus erinaceus, Parkia biglobosa, Afzelia africana, Khaya senegalensis et Vitex doniana. Oloukoi et al. (2006) soulignent aussi la destruction de la végétation principalement par trois acteurs : les agriculteurs pratiquant l'écobuage ; les producteurs de charbon ; les éleveurs transhumants émondant et élaguant des essences pour l'alimentation de leur bétail. Plusieurs auteurs tels que N'Guessan et al. (2006) et Diallo et al. (2010), respectivement en Côte d'Ivoire et au Mali, suggèrent que les coupes abusives de bois représentent le facteur déterminant de la dynamique régressive des espèces végétales ligneuses et donc d'un changement de classes d'occupation du sol. En outre, la facilité de pénétration dans ces différents milieux forestiers par des migrants agricoles venus des zones surpeuplées (sud du Bénin) ou semi-arides (nord-ouest du Bénin) peut expliquer leur vulnérabilité à l'anthropisation (IFN, 2007). La savanisation résultant de la dégradation quasi totale des ressources forestières liée aux activités humaines confirme bien les résultats de Judex (2008). La production des tubercules d'igname serait l'une des causes principales de la déforestation consécutive aux activités agricoles. Selon Adanguidi (2001), l'igname joue un rôle essentiel dans l'alimentation, le commerce et surtout la vie socioculturelle des populations. Cependant, l'igname est une culture très exigeante en main-d'œuvre et en fertilité (Inrab, 2001). Le reste de la production agricole cotonnière est assuré dans les savanes comme dans la plupart des pays tropicaux, où ce sont particulièrement des parcelles de petite taille qui sont l'objet principal du déboisement. 


\section{Références bibliographiques}

\section{Influence de la déforestation et de la savanisation sur la dynamique du paysage}

Sous l'influence des activités socio-économiques, la configuration et la composition de l'occupation des sols dans la zone d'étude ont changé. La forte pression anthropique qui se manifeste par des prélèvements incontrôlés, surtout à des fins de carbonisation et de mise en culture (Adjonou et al., 2009), a dégradé la végétation et les paysages. Plusieurs auteurs reconnaissent que les massifs forestiers sont devenus un des écosystèmes les plus dégradés dans les zones soudano-guinéennes. La dégradation de ces massifs forestiers a porté tant sur leur surface ou leur nombre que sur leur type (Barima et al., 2009). Les formations forestières dominantes en 1972 ont été largement transformées en formations savanicoles puis en mosaïque champs-jachères. Ainsi, ces forêts, qui constituaient initialement la matrice dominante du paysage, sont devenues des fragments d'habitats. Faute de connectivité, la diminution de la taille de ces fragments et l'augmentation de leur isolement réduisent, à long terme, la viabilité des populations d'espèces végétales et animales qui y vivent par la limitation, voire la disparition des échanges entre ces populations (Cristofoli et Mahy, 2010).

\section{Conclusion}

La présente étude met en évidence la pertinence de l'approche cartographique à partir des images satellitaires et contribue ainsi à l'interprétation de la dynamique paysagère dans le centre du Bénin. Elle révèle que les pressions humaines actuelles sur les ressources forestières sont en rupture avec les capacités de régénération des formations végétales naturelles qui sont ainsi sérieusement menacées. En outre, une extension rapide des zones à emprise agricole est observée au détriment des formations boisées denses. Ce mouvement global, partant des noyaux d'agglomération et hameaux ruraux, associé à une intensification des activités humaines, questionne la durabilité des processus écologiques, économiques et sociaux dans une zone fortement dépendante des conditions climatiques. Il semble donc urgent de développer une stratégie de gestion intégrée et participative aussi bien à l'échelle locale que régionale afin de préserver de façon durable les ressources naturelles. Les principes de cette gestion doivent reposer sur l'élaboration concertée, entre décideurs locaux et populations, de plans d'aménagement prenant en compte la définition claire des espaces agricoles, la protection des zones naturelles ainsi que la maîtrise des flux et processus écologiques dont ces zones font l'objet.
ABDEL-KAWY O. R., ROD J. K., ISMAIL H. A., SULIMAN A. S., 2011. Land use and land cover change detection in the western Nile delta of Egypt using remote sensing data. Applied Geography, 31 (2): 483-494.

ADAM S. K., BOKO M., 1993. Le Bénin. Nouvelle édition. Cotonou, Bénin, Éditions du Flamboyant-Edicef, 93 p.

ADANGUIDI J., 2001. Réseaux, marchés et courtage : la filière igname au Bénin (1990 à 1997). Thèse, Université de Hohenheim, Allemagne, $301 \mathrm{p}$.

ADJONOU K., BELLEFONTAINE K. R., KOKOU K., 2009. Les forêts claires du Parc national Oti-Kéran au Nord-Togo : structure, dynamique et impacts des modifications climatiques récentes. Sécheresse, 20 (1) : 1-10.

AFOUDA F., 1990. L'eau et les cultures dans le Bénin central et septentrional : étude de la variabilité des bilans de l'eau dans leurs relations avec le milieu rural de la savane africaine. Thèse, Université de Paris IV (Sorbonne), France, 448 p.

BARIMA Y. S. S., BARBIER N., BAMBA I., TRAORE D., LEJOLY J., BOGAERT J., 2009. Dynamique paysagère en milieu de transition forêt-savane ivoirienne. Bois et Forêts des Tropiques, 299 (1) : 15-25.

BOGAERT J., CEULEMANS R., SALVADOR-VAN EYSENRODE D., 2004. Decision tree algorithm for detection of spatial processes in landscape transformation. Environmental Management, 33 (1): 62-73.

CALOZ R., LAZER T. J., WILLEMIN G., 1993. Création d'une orthoimage à l'aide d'un modèle numérique d'altitude : influences des modes de rééchantillonnage radiométrique. In : Dubois J.-M. M., Cavayas F., Lafrance P. (dir.). Télédétection appliquée à la cartographie thématique et topographique. Quatrièmes journées scientifiques du Réseau Télédétection de l'UREF, Montréal, 21-23 octobre 1991. Québec, Canada, Presses de l'Université du Québec, 17-30.

CALOZ R., COLLET C., 2001. Précis de télédétection. Volume 3 : Traitements numériques d'images de télédétection (Universités francophones). Québec, Canada, Presses Polytechniques du Québec, 368 p.

COPPIN P., JONCKHEERE I., NACKAERTS K., MUYS B., LAMBIN E., 2004. Digital change detection methods in ecosystem monitoring: a review. International Journal of Remote Sensing, 25 (9): 1565-1596.

CRISTOFOLI S., MAHY G., 2010. Restauration écologique : contexte, contraintes et indicateurs de suivi. Biotechnologie, Agronomie, Société et Environnement, 14 (1) : 203-211. 
DIALLO H., BAMBA I., BARIMA S. S. Y., VISSER M., BALLO A., MAMA A., VRANKEN I., MAIGA M., BOGAERT J., 2010. Effets combinés du climat et des pressions anthropiques sur la dynamique évolutive de la végétation d'une zone protégée du Mali (Réserve de Fina, Boucle du Baoulé). Sécheresse, 22 (8) : 97-107.

DIRZO R., GARCIA M. C., 1992. Rates of Deforestation in Los Tuxtias, a Neotropical Area in Southeast Mexico. Society for Conservation Biology, 6 (1): 84-90.

FOODY G. M., 2002. Status of land cover classification accuracy assessment. Remote Sensing of Environment, 80: 185-201.

GALICIA L., GARCIA-ROMERO A., 2007. Land use and land cover change in Highland temperate forests in the Izta-Popo national park, central Mexico. Mountain Research and Development, 27 (1): 48-57.

HOUNTONDJI Y. H., 2008. Dynamique environnementale en zone sahélienne et soudanienne de l'Afrique de l'Ouest : analyse des modifications et évaluation de la dégradation du couvert végétal. Thèse, Université de Liège, Belgique, $113 \mathrm{p}$.

IFN, 2007. Rapport d'exécution de l'Inventaire Forestier National (IFN), Projet Bois de Feu, phase II. Cotonou, Bénin, ministère de l'Environnement et de la Protection de la Nature, 41 p.

IGN France, 1969. Carte de l'Afrique de l'Ouest à 1/200 000. République du Dahomey. Feuilles NC-31-II, NC-31-III, NB-31XX-XXI.

IGUE A. M., 2000. The use of a soil and terrain database for land evaluation procedures: Case study of Central Benin. Thèse, Université de Hohenheim, Allemagne, 235 p.

INRAB, 2001. Recherche agricole pour le développement. Rapport annuel. Cotonou, Bénin, Institut national de recherche agronomique du Bénin, $70 \mathrm{p}$.

JUDEX M., 2008. Modelling of the land use dynamics in central Benin with the Xulu framework. Thèse, Universite de Bonn, Allemagne, $130 \mathrm{p}$.

JUSOFF K., SETIAWAN I., 2003. Quantifying deforestation in a permanent forest reserve using vectorised Landsat TM. Journal of Tropical Forest Science, 15: 570-582.

KHORRAM S., BIGING G. S., CHRISMAN N. R., COLBY D. R., CONGALTON R. G., DOBSON J. E., FERGUSON R. L., GOODCHILD M. F., JENSEN J. R., MACE T. H., 1999. Accuracy assessment of remote sensing-derived change detection. Bethesda (MD), USA, The American Society for Photogrammetry and Remote Sensing, $65 \mathrm{p}$.

MAS J. F., 2000. Une revue des méthodes et des techniques de télédétection du changement. Canadian Journal of Remote Sensing, 26 (4) : 349-362.
MAYAUX P., EVA H., PALUMBO I., GRÉGOIRE J.-M., FOURNIER A., SAWADOGO L., 2007. Apport des techniques spatiales pour la gestion des aires protégées en Afrique de l'Ouest. In : Fournier A., Sinsin B., Mensah G. A. (éds). Quelles aires protégées pour l'Afrique de l'Ouest ? Conservation de la biodiversité et développement. Paris, France, IRD, coll. Colloques et séminaires, 321-328.

N'GUESSAN E., DIBI N'DAH H., BELLAN M.-F., BLASCO F., 2006. Pression anthropique sur une réserve forestière en Côte d'Ivoire : Apport de la télédétection. Télédétection, 5 (4) : 307-323.

OLOUKOI J., MAMA V. J., AGBO F. B., 2006. Modélisation de la dynamique de l'occupation des terres dans le département des Collines au Bénin. Télédétection, 6 (4) : 305-323.

PHAM T. T. H., BONN F., DUBOIS J.-M., 2007. Démarche méthodologique pour la détection des changements d'un milieu morcelé en utilisant des images à moyenne résolution spatiale : application à une région littorale au Viêt-Nam. Télédétection, 7 (5) : 302-323.

PONTIUS R. G., 2000. Quantification error versus location in comparison of categorical maps. Photogrammetric Engineering and Remote Sensing, 66 (8): 1011-1016.

ROBIN M., 2002. Télédétection : des satellites au SIG. Une analyse complète du processus de création d'un type essentiel d'information géographique, $2^{\mathrm{e}}$ édition, Paris, France, Nathan Université, 318 p.

SCIENTIFIC COUNCIL FOR AFRICA SOUTH OFTHE SAHARA, 1962. Réunion de spécialistes du CSA en matière de phytogéographie. CSA Specialist Meeting on Phytogeography. Yangambi, Congo Belge, 28 juillet-8 août 1956. Yangambi, CCTA, 35 p.

TABOPDA W. G., FOTSING J.-M., 2010. Quantification de l'évolution du couvert végétal dans la réserve forestière de LafMadjam au nord du Cameroun par télédétection satellitale. Sécheresse, 21 (3) : 169-178.

TREPANIER I., DUBOIS J. M. M., BONN F., 2002. Suivi de l'évolution du trait de côte à partir d'image HRV (XS) de SPOT : delta du fleuve Rouge, Viêt-Nam. International Journal of Remote Sensing, 23 (5): 917-937.

TCHIWANOU M., 2001. Étude prospective du secteur forestier en Afrique (FOSA), République du Bénin. FAO/EC. Point FocalBénin, 32 p. 


\section{Bois et forêts des tropiques Note to authors}

\section{Please help us to optimise processing of your files and folders!}

When you submit a draft article, you must give us e-mail address of each author, please send the files by email or $\mathrm{ftp}$ transfer to the BFT journal address. If necessary, you may send the printable version on CD-Rom to us by post.

You will need to send us 3 kinds of files: - File 1 containing your text, abstracts and tables;

- File 2 containing illustrations (photographs and figures with low resolution) and legends, with source files preferably in very high resolution or vector mode;

- File 3 (group of files) containing illustrations and photographs (and the name of the photograph's author in each case).

For figures, diagrams and maps, please use vector software that allows files to be opened with Illustrator, saving them in eps format only. For charts, add files from spreadsheet programs. High-quality print originals of all files must also be supplied, as the use of electronic files cannot be guaranteed. Photo files in $10 \mathrm{~cm} \times 15 \mathrm{~cm}$ format, with a minimum resolution of $300 \mathrm{dpi}$, must be compressed into JPEG at maximum quality. If you cannot provide a digital version of your photographs, you may send us prints or slides.

Articles must be no longer than $\mathbf{6} \mathbf{5 0 0}$ words (35000 signs including spaces), not counting the abstract. You may choose to send your articles in English or French, with an abstract and six key words in the same language as the article, at least. The abstracts will be translated and subsequently revised by the editor.

For further information, please contact: CIRAD, C-DIR/B

Bois et forêts des tropiques

Campus international de Baillarguet

34398 Montpellier Cedex 5

France

E-mail: bft@cirad.fr

bft.cirad.fr

\section{Articles:}

Articles must present meaningful results that are potentially transferable to other fields.

$\mathbf{6 5 0 0}$ words (text + tables + references) with a minimum of 4 photos with legends plus a 275-word abstract.

\section{Research notes:}

Short articles concerning studies that are nearing completion, to present preliminary results and/or research prospects. These may, in particular, present a recent or forthcoming thesis. $\mathbf{2 5 0 0}$ words (text + tables + references) with a minimum of 3 photos with legends plus a 275-word abstract.

Articles must be presented in the following order:

- First page containing the title (maximum 10 words), first names, surnames and addresses of the authors, in full. For the first-named author or the author corresponding with the journal, please provide a current professional address and email address.

- Text in Times New Roman, size 12, with 1.5 line spacing and numbered pages; - List of bibliographic references (maximum 20);

- Abstract containing no more than $\mathbf{2 7 5}$ words;

- The legends to illustrations (maximum 6 figures, maps and/or diagrams) and photos (about 10) must be supplied on a separate page. Sources or credits for all illustrations must be mentioned as required. The author of each photograph must be named.

- Tables (MAXıмum 5), each with a legend;

- Photos must be numbered in the order in which they appear in the text.

The text should be composed in a single font. Latin names must be in italics, and the names of authors cited in the text in small caps, e.g. Tchoume et al. All acronyms and abbreviations must be given in full the first time they appear in the text (or as an end-ofpage note).
All tables, figures and photographs must have a legend and must be numbered in the order in which they appear in the text. Tables, figures and photos must be announced in the text as follows: tables in Roman numerals (e.g. Table V), figures and photos in Arab numerals (e.g. Figure 2, Photo 2). For figures, please supply high-quality print-outs and make sure you have obtained any necessary permission to publish them.

\section{Bibliographic references must be written as follows in the text:}

DIOUF (2001); DOMMERGUES, MANGENOT (1970); (POUTOULI, 1994; CADOU et al., 1952), and when there are three or more authors: ROSSMAN et al. (1995). The references given in the list only concern publications mentioned in the text. They are listed in alphabetical order by author. They are presented as follows:

Book: IBGE, 1998. Produçáo da extraçáo vegetal e da silvicultura 1995. Rio de Janeiro, Brazil, IBGE, vol. 10, 277 p.

Paper given at a conference: BERTRAND A., BABIN D., WEBER J., 2000. Pauvreté et gestion des ressources renouvelables dans les pays en développement: les liaisons dangereuses? In: Forests and society: The role of research, Vienna, Austria, 8 July-8 August 2000. Kuala Lumpur, Malaysia, IUFRO, 244-245.

Article in a periodical: BHAT K. M. M., 2000. Non-destructive techniques for wood quality assessment of plantation-grown teak. Bois et Forêts des Tropiques, 263 (1): 6-16.

Thesis: PETIT S., 2000. Environnement, conduite des troupeaux et usage de l'arbre chez les agropasteurs peuls de l'Ouest burkinabé. Thesis, Orleans University, France, 528 p. 